\title{
Diseño y metodología de la Encuesta Nacional de Salud 2000
}

\author{
Jaime Sepúlveda, MD, M en C, D en C,(1) Roberto Tapia-Conyer, $\mathrm{PhD},{ }^{(2)}$ OscarVelásquez,† MC, ${ }^{(3)}$ \\ José Luis Valdespino, MC, MSP, ${ }^{(4)}$ Gustavo Olaiz-Fernández, MC, MSP, ${ }^{(2)}$ Pablo Kuri, MC, ${ }^{(2)}$ \\ Elsa Sarti, PhD, ${ }^{(3)}$ Carlos J Conde-González, QBP, M en C, D en C.(I)
}

\section{Sepúlveda J,Tapia-Conyer R, Velásquez O,Valdespino JL, Olaiz-Fernández G, Kuri P, Sarti E, Conde-González C. Diseño y metodología de la Encuesta Nacional de Salud 2000. Salud Publica Mex 2007;49 supl 3:S427-S432.}

\begin{abstract}
Resumen
Objetivo. Como parte del Sistema de Encuestas Nacionales de Salud, durante los últimos meses de 1999 y los primeros tres del año 2000 se realizó la Encuesta Nacional de Salud de México (ENSA 2000). Se estudió la accesibilidad, calidad, utilización y cobertura de los Servicios de Salud; de modo adicional se actualizaron los marcadores serológicos de enfermedades infecciosas prevenibles por vacunación, infecciones de transmisión sexual y hepatitis. Material y métodos. Para la ENSA 2000 se seleccionaron tres grupos etarios y a los utilizadores de los servicios de salud. Se captó la información mediante entrevista directa y se tomaron muestras biológicas para análisis clínicos y medidas de parámetros biológicos y somatométricos. El diseño muestral de la ENSA 2000 fue probabilístico, polietápico, estratificado y de conglomerados. El tamaño de la muestra fue de I 470 viviendas por estado, para un total de 47040 viviendas a nivel nacional; los factores de expansión se modificaron por la falta de respuesta y la posestratificación. El personal operativo se capacitó y estandarizó para mantener una alta respuesta, en especial para las muestras de sangre. Resultados. En total se obtuvieron 83157 muestras de sangre de las 94000 esperadas (respuesta de $88 \%$ ) que se mantuvieron refrigeradas en tanto se ubicaron en el laboratorio del Instituto Nacional de Salud Pública donde se prepararon cuatro alícuotas y se congelaron a $-150^{\circ} \mathrm{C}$ hasta el análisis.
\end{abstract}

Palabras clave: metodología; análisis demográfico; análisis transversal; México
Sepúlveda J,Tapia-Conyer R,Velásquez O,Valdespino JL, Olaiz- Fernández G, Kuri P, Sarti E, Conde-González C. National Health Survey 2000: design and methodology. Salud Publica Mex 2007;49 suppl 3:S427-S432.

\begin{abstract}
Objective. The 2000 Mexican National Health Survey (NHS) was created as part of the System for National Health Surveys conducted during the last months of 1999 and the first three of 2000. The 2000 NHS is a probabilistic survey of households from which users of health services were selected according to three age groups. Information was gathered through direct interviews with appropriate informants. Biological samples were taken for clinical tests as well as for measuring biological and somatometric parameters. Material and Methods. The sample design of the $2000 \mathrm{NHS}$ was stratified and clustered. Sample size was I 470 households per state for a total of 47040 households nationwide (there are 32 states in México). Sample weights were calculated and modified according to the non-response and post-stratified to calibrate for population distribution. Interviewers were trained in order to maintain a high response rate, especially for biological samples. Results. A total of 83157 blood samples were collected from the 94000 expected (88\% response rate). All samples were refrigerated immediately after collection and divided in four vials for storage at the National Institute for Public Health's laboratory. Samples were frozen at $-150^{\circ}$ Celsius until further analysis.
\end{abstract}

Key words: methodology; demographic analysis; cross-sectional analysis; Mexico

(I) Instituto Nacional de Salud Pública de México. México

(2) Secretaría de Salud. México.

(3) Centro Nacional de Vigilancia Epidemiológica y Control de Enfermedades. México.

(4) Instituto Nacional de Salud Pública; actualmente en el Laboratorio de Biológicos y Reactivos de México (Birmex).

Fecha de recibido: 28 de febrero de 2006 • Fecha de aceptado: 7 de febrero de 2007 Solicitud de sobretiros: Dr. Gustavo Olaiz Fernández. Instituto Nacional de Salud Pública. Av. Universidad 655, Col. Santa María Ahuacatitlán. 62508 Cuernavaca, Morelos, México.

Correo electrónico: golaiz@insp.mx 
Como preludio del establecimiento de un Sistema UNacional de Encuestas de Salud, en 1986 se aplicó en México la primera Encuesta Nacional de Salud (ENSA I). ${ }^{1}$ A partir de entonces se han efectuado de manera sistemática; cabe destacar por orden cronológico la realización en 1987 de la Encuesta Nacional Seroepidemiológica I(ENSE I), ${ }^{2}$ la Encuesta Nacional de Nutrición I (ENN I); ${ }^{3}$ en 1988, la Encuesta Nacional de Adicciones 1(ENAI), ${ }^{4}$ en 1992, la Encuesta Nacional de Cobertura de Vacunación (ENCOVA); ${ }^{5}$ en 1993, la Encuesta Nacional de Enfermedades Crónicas (ENEC), ${ }^{6}$ así como la ENA II ${ }^{7}$ en 1994, la segunda edición de la ENSA, ${ }^{8}$ la cual estuvo orientada al estudio de la accesibilidad, la calidad, la utilización y la cobertura de los servicios de salud; y en 1998, la tercera edición de la ENA y la segunda de la ENN. ${ }^{9}$ Los resultados de las encuestas son de importancia para evaluar el cumplimiento de las metas de los programas prioritarios y las medidas diseñadas para su instrumentación, además de los nuevos programas de enfermedades crónicas y degenerativas una vez puesta la encuesta en marcha.

\section{Objetivos}

Los objetivos de la Encuesta Nacional de Salud 2000 (ENSA 2000) fueron los siguientes:

1. Calcular la frecuencia y la distribución de los indicadores positivos de salud, factores de riesgo, enfermedades (agudas y crónicas), lesiones y discapacidad en el ámbito nacional.

2. Cuantificar la seroprevalencia de los marcadores de factores de riesgo y anticuerpos contra enfermedades específicas.

3. Precisar las prevalencias estatales de diabetes mellitus, hipertensión arterial, obesidad, dislipidemias y padecimientos concomitantes.

4. Contribuir en la evaluación de programas de salud (p. ej., vacunación, detección de cáncer cervicouterino y otros).

5. Propiciar el conocimiento y la identificación de factores genéticos, ambientales, socioeconómicos, culturales y de estilos de vida relacionados con la salud y los padecimientos estudiados.

6. Identificar factores vinculados con la utilización de los servicios de salud.

7. Calcular la magnitud del gasto de bolsillo atribuido a la atención a la salud.

8. Cuantificar los indicadores de calidad de los servicios de salud.

\section{Bases metodológicas}

Los requerimientos de información actualizada para el sector salud y el análisis de las encuestas previas del Sistema de Encuestas de Salud fueron el punto de partida para determinar la temática a incluir en la ENSA 2000, sus definiciones, la formulación de preguntas y el diseño de los instrumentos de captación.

En reuniones con especialistas en los temas y usuarios de la información se pusieron a consideración los cuestionarios, el diseño de la muestra y la propuesta operativa.

Las bases metodológicas de la ENSA 2000 son las siguientes:

1. Es una encuesta por muestreo probabilístico, polietápico, estratificada y por conglomerados.

2. Se definió como una encuesta que cubriera los hogares de viviendas ; se consideró a todos sus integrantes y y de ellos se seleccionaron las siguientes subpoblaciones:

- Utilizadores de servicios de salud, ambulatorios u hospitalarios.

- Un niño de 0 a 9 años de edad.

- Un adolescente de 10 a 19 años de edad.

- Un adulto de 20 o más años de edad.

3. Se captó la información recogida en una entrevista directa al informante adecuado y se tomaron análisis clínicos y parámetros biológicos.

4. El periodo de levantamiento fue de seis meses y medio, de noviembre de 1999 a junio de 2000.

5. Capacitación, en dos fases, con una duración total de cuatro semanas.

6. La operación se adecuó al manejo, conservación, traslado y concentración de las muestras de orina y sangre en el laboratorio del Instituto Nacional de Salud Pública (INSP).

7. Sistema inteligente de captura, desarrollo de rutinas y validación de los archivos, además de la creación de un banco de datos.

\section{Diseño conceptual general}

La Encuesta Nacional de Salud 2000 recolectó información relacionada con las características geográficas de las localidades y entidades federativas; para ello se utilizaron las bases de datos georreferenciales existentes en el país, como las del Instituto Nacional de Estadística, Geografía e Informática (INEGI) y del Instituto de Inves- 
tigaciones Geográficas (IIG) de la Universidad Nacional Autónoma de México (UNAM), entre otras.

En el diseño conceptual de la encuesta se consideraron como unidades de análisis, en primer término, las viviendas, y se asignó el papel de informante al responsable del hogar; en segundo lugar se incluyó a los demás integrantes y, entre ellos, a los que habían utilizado servicios de salud ambulatorios u hospitalarios en los 12 meses anteriores a la entrevista.

En el interior de cada hogar se seleccionó de manera aleatoria a los siguientes individuos: a) un niño de cero a nueve años de edad (los informantes próximos fueron la madre o el responsable del niño); b) un adolescente de 10 a 19 años de edad; y c) un adulto de 20 o más años de edad. Estos últimos constituyeron las unidades de análisis para el estudio de la prevalencia de factores de riesgo, enfermedades agudas y crónicas, y daños a la salud por accidentes o violencias. En todos los casos se obtuvo el consentimiento informado de los entrevistados, de acuerdo con los principios éticos para la investigación médica en seres humanos de la Declaración de Helsinki. En consecuencia, se notificaron a los entrevistados sus derechos como sujetos de la encuesta y la seguridad de que se conservaría el anonimato. Por razones operativas no se solicitó que firmaran los formatos de consentimiento, pero en todos los casos los supervisores verificaron que se llevara a cabo. De forma adicional, el INSP hizo un llamado en los medios para que la población despejara cualquier duda o mostrara interés en ser excluida del estudio.

\section{Organización del banco de muestras biológicas}

En la Encuesta Nacional de Salud 2000 se colectaron 83157 muestras de sueros humanos; el diseño esperaba un máximo de 94000 muestras, lo que resultó en una proporción de $88 \%$ de respuesta a sangre. Además, se cuenta con 43560 muestras de paquetes de glóbulos blancos para extracción de DNA de los 47000 esperados (respuesta de 92\%). Los sueros provienen de personas de ambos sexos comprendidas desde la infancia hasta la senectud y las células blancas se obtuvieron de adultos de ambos sexos.

Las muestras de sangre se colectaron en los domicilios, previo consentimiento informado. Una muestra se obtuvo de niños, adolescentes y adultos mediante venopunción al vacío con tubos Vacutainer SST con gel separador (Becton-Dickinson). Una segunda muestra se obtuvo sólo de adultos, con tubos Vacutainer heparinizados (Becton-Dickinson) con el fin de separar el plasma y luego recuperar por aspiración la capa de células blancas (alrededor de $0.5 \mathrm{ml}$ ), la cual se mezcló con $0.5 \mathrm{ml}$ de solución reguladora de $\mathrm{pH} 8$ Tris- $\mathrm{HCl}$ (10 mM)- $\mathrm{NaCl}(400 \mathrm{mM})$-EDTA (25 mM)-SDS (0.5\%) en crioviales de $2 \mathrm{ml}$. Los sueros y los paquetes globulares así obtenidos se almacenaron en refrigeración a $4^{\circ} \mathrm{C}$, por no más de cinco días, y trasladados refrigerados al laboratorio del banco de sueros y DNA del INSP. Con cada suero obtenido se prepararon cuatro alícuotas de $1 \mathrm{ml}$ en crioviales de $2 \mathrm{ml}$ de capacidad.

La colección completa de ambos tipos de muestras se conserva en el banco de sueros y DNA en las instalaciones del INSP. En este banco, las muestras biológicas se mantienen en cámaras de congelación automatizadas con vapores de nitrógeno líquido a $-150^{\circ} \mathrm{C}$.

\section{Características de los hogares y la población}

Las características generales más relevantes de la población estudiada en la ENSA 2000 corresponden a dos apartados: las variables sociodemográficas y las características de la vivienda. Los cuestionarios utilizados se han distribuido de manera amplia.

\section{Necesidades de salud}

Antes de que una persona emplee los servicios de salud, debe percibir que los necesita; por consiguiente, la necesidad se considera la causa inmediata del empleo de los servicios, que puede variar de acuerdo con factores predisponentes y habilitadores. Es posible su cuantificación a partir de la percepción del estado de salud, la frecuencia del dolor, síntomas, días de actividad perdidos, discapacidad, gravedad percibida de un problema, etc., ${ }^{10-12}$ (figura 1).El capítulo sobre necesidades de salud incluye percepción de la propia situación de salud; prevalencia por lapsos de las enfermedades agudas y crónicas; daños a la salud originados a partir de accidentes, violencias o adicciones; prevalencia de discapacidad; mediciones de peso y talla; tensión arterial; y obtención de muestras biológicas.

\section{Utilización de los servicios de salud}

En teoría existe una correspondencia entre la necesidad de salud percibida por la población y el nivel de utilización de los servicios. Ante dicha necesidad se activan mecanismos con el fin de satisfacerla, según su cultura, poder de compra y personalidad. Desde la perspectiva poblacional, la utilización guarda relación con la percepción del estado de salud o la enfermedad que presente la población, las decisiones de los usuarios y los proveedores de salud. ${ }^{11,13}$

También intervienen factores habilitadores y predisponentes en la utilización de los servicios de salud. Para la 


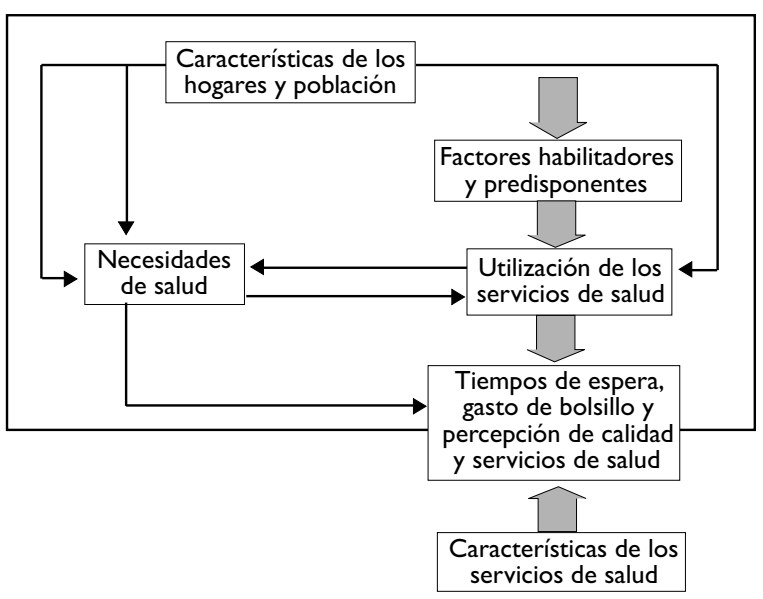

Figura I. Contenido del diseño conceptual de los Cuestionarios de Hogar y Población y el de Utilización de servicios de salud. Encuesta Nacional de Salud 2000, MÉXICO

disposición de éstos se considera el grado de ajuste entre las características de la población y las de los recursos en el proceso de búsqueda y obtención de la atención de salud.

Los encuestados refieren tiempos de espera y traslado, gastos de bolsillo y percepción de la calidad para obtener atención de servicios de salud ambulatoria, además de los gastos de bolsillo consumidos en la consulta ambulatoria y la hospitalización, que provienen del propio usuario. Por otra parte, la calidad de la atención se vincula con el grado de mejoría esperada en el nivel de salud atribuible a la atención recibida. Para el análisis de esta variable se consideraron tres dimensiones: ${ }^{14}$

1. Estructura. Incluye los recursos humanos, materiales y financieros. Para el diseño de la encuesta sólo se tomaron en cuenta los recursos materiales.

2. Proceso. Se consideró lo que médicos y otros proveedores de los servicios de salud hacen por los pacientes, incluida la capacidad para otorgar la atención, además de las acciones que los individuos realizaron para sí mismos.

3. Resultado. En esta dimensión se valoró todo aquello que se obtiene para el paciente en términos de un cambio del estado de salud, ya sea de manera positiva o negativa y que puede atribuirse a la atención.

\section{Diseño muestral}

El diseño muestral de la ENSA 2000 fue probabilístico, polietápico, estratificado y de conglomerados. Para garantizar que las estimaciones obtenidas por la encuesta tuvieran calidad aceptable, fue necesario que el tamaño de la muestra definida para cada parámetro de interés fuera suficiente. Por ello, el cálculo de dicho tamaño se relacionó con los parámetros a estimar, el nivel de confianza y el error relativo máximo aceptable. Más aún, fue necesario incluir una corrección por falta de respuesta y el efecto de diseño. ${ }^{15}$

En consecuencia, el tamaño se calculó mediante la fórmula:

$$
n=\frac{Z_{\alpha / 2}^{2}(1-P) \text { deff }}{r^{2} P T R}
$$

donde:

$n=\quad$ tamaño de la muestra

$P=\quad$ proporción a estimar $(p=0.05)$

$Z_{\alpha / 2}^{2}=$ cuantil de una distribución normal relacionado con un nivel de confianza deseado $\left(Z_{\alpha / 2}^{2}=\right.$ 1.96)

$R=\quad$ error relativo máximo aceptable $(r=0.3)$

deff = efecto del diseño, que es la pérdida o ganancia en la eficiencia de diseño, por tratarse de uno complejo $(\operatorname{def} f=3)$

$T R=\quad$ tasa de respuesta $(T R=70 \%)$

Por consiguiente, el tamaño de la muestra fue de 1473 viviendas por estado. Este tamaño se redondeó a 1 470, lo que arrojó 47040 viviendas a escala nacional.

\section{Esquema de selección}

Los pasos del proceso de selección fueron los siguientes:

- Asignación proporcional de viviendas en muestra por estrato urbano-rural.

- Selección de 14 municipios por estado, con reemplazo y probabilidad proporcional de número de viviendas en él.

- $\quad$ Selección de cinco AGEB por municipio, con probabilidad proporcional al tamaño.

- $\quad$ Selección de tres manzanas por AGEB, con igual probabilidad.

- $\quad$ Selección de siete viviendas por manzana, con la misma probabilidad.

- Selección de un individuo en el grupo de edad de interés, con igual probabilidad. 


\section{Factores de expansión}

De acuerdo con el esquema de selección de la muestra, las probabilidades de selección de una vivienda se calculan como sigue:

$$
P\left(V_{h i}\right)=\frac{n_{h} \cdot 5 \cdot 21}{N_{h}}
$$

donde:

$h=\left\{\begin{array}{l}0 \text { si el estrato es rural } \\ 1 \text { si el estrato es urbano }\end{array}\right.$

$\mathrm{n}_{\mathrm{h}}=$ número de municipios seleccionados en el estrato $h$, para $h=0,1$

$\mathrm{N}_{\mathrm{h}}=$ número de viviendas habitadas en el estrato $h$

Por otro lado, la probabilidad de selección de un individuo perteneciente al grupo de edad de interés es la siguiente:

$P\left(I_{h i j}=P\left(V_{h i}\right) \cdot \frac{1}{H_{h i g}}=\frac{n_{h} \cdot 5 \cdot 21}{N_{h}} \cdot \frac{1}{H_{h i g}}\right.$

donde:

$h=\left\{\begin{array}{l}0 \text { para el grupo de edad de } 0 \text { a } 9 \text { años } \\ 1 \text { para el grupo de edad de } 10 \text { a } 19 \text { años } \\ 2 \text { para el grupo de edad de } 20 \text { años y más }\end{array}\right.$

$H_{\text {hig }}=$ número de individuos en la vivienda $i$-ésima del estrato $h$ y perteneciente al grupo de edad $g=0$, 1,2

De esta manera, el factor de expansión es:

$$
F_{h i}=\frac{N_{h}}{n_{h} \cdot 5 \cdot 21}
$$

Si se desea obtener cálculos sobre características de los individuos se debe utilizar el factor:

$$
F_{h i g}=\frac{N_{h} \cdot H_{h i g}}{n_{h} \cdot 5 \cdot 21}
$$

\section{Ajuste por falta de respuesta y posestratificación}

Los factores de expansión se multiplicaron por dos factores adicionales. El primero de ellos, referido como $A_{1(\text { hig) }}$ se incluyó para ajustar el efecto de la falta de respuesta. De esta manera, el factor de expansión con el ajuste por falta de respuesta es:

$$
F_{h i g}=A_{1(h i g)} \cdot F_{h i g}
$$

El segundo es un elemento para calibrar los factores de expansión del diseño muestral. Los factores de expansión calibrados se usan con frecuencia para mejorar las cuantificaciones obtenidas a partir de una encuesta, mediante el uso de información auxiliar. ${ }^{16}$ En el caso de la ENSA 2000, los pesos calibrados se utilizaron para ajustar el efecto de subrepresentación o sobrerepresentación de ciertos grupos y permitir los cálculos de totales actualizados según las cifras del XII Censo de Población y Vivienda 2000. El método de calibración empleado aparece en el trabajo de $\operatorname{Lee}^{17} \mathrm{y}$ se conoce como pos-estratificación.

Si se denota por $A_{2(h i g)}$ el factor de calibración, el factor de expansión final es:

$$
\overline{\bar{F}}_{\text {hig }}=A_{2(h i g)} \cdot \bar{F}_{h i g}=A_{1(h i g)} \cdot A_{2(h i g)} \cdot F_{h i g}
$$

Cabe señalar que para aquellos grupos subrepresentados el factor $A_{2(h i g)}$ es mayor a la unidad, mientras que para los grupos sobrerrepresentados es menor a uno. Es decir, los individuos de grupos subrepresentados tienen un ponderador mayor que los individuos de grupos sobrerepresentados.

De lo anterior se observa que el esquema de muestreo no es autoponderado, lo que significa que cada vivienda en la muestra representa un número diferente de viviendas. Por lo tanto, cualquier indicador o tabulación requiere para su construcción el uso de los factores de expansión.

\section{Medidas operativas}

La captación de la información se llevó a cabo en ocho etapas que comprendieron el levantamiento simultáneo de datos en cinco entidades en general, excepto en la última que sólo abarcó al Distrito Federal. El tiempo promedio de permanencia en una entidad fue de 23 días.

Con la finalidad de cumplir con los objetivos de la ENSA 2000 se contrató a personal de enfermería en todos los niveles de la estructura operativa. A cada una de las entidades se asignaron cuatro supervisores. En cada entidad se formaron 20 equipos integrados por dos entrevistadores; cada supervisor tuvo a su cargo cinco equipos que distribuyó en dos vehículos (figura 2).

En promedio se tuvieron una carga de trabajo diaria de cuatro viviendas por equipo; dos horas por entrevista; y tres visitas a cada vivienda. Al finalizar la jornada diaria, cada equipo entregaba los cuestionarios y las muestras biológicas a los supervisores, para que ellos revisaran y prepararan los cuestionarios para su envío a la Ciudad de México. ${ }^{18}$ 


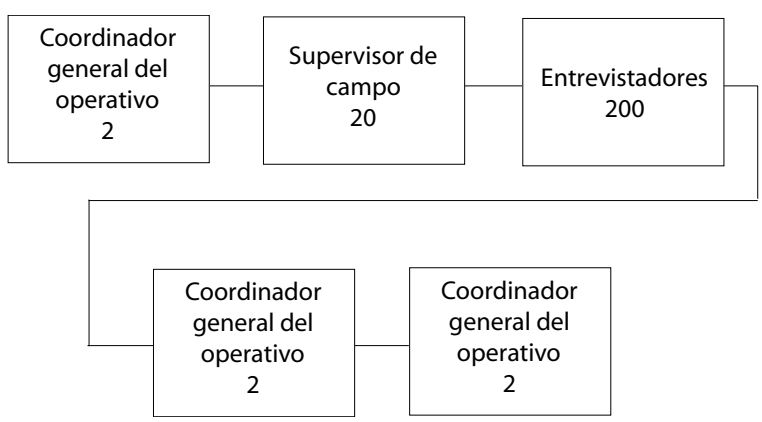

Figura 2. Estructura operativa de la Encuesta NacioNal de Salud 2000, México

\section{Capacitación}

Para garantizar la calidad y veracidad de los datos se llevó a cabo un programa de capacitación en dos fases, de modo que se aseguró la estandarización y control de los procedimientos para la obtención de muestras biológicas, toma de medidas somatométricas, así como el manejo y llenado adecuado de los cuestionarios.

El periodo de capacitación fue de 30 días, dividido en dos etapas. La primera comprendió el uso de cuestionarios y el procedimiento y estandarización para la obtención de muestras y medidas antropométricas, con una duración de 20 días.

La segunda etapa se cumplió después de la prueba piloto, una vez ajustados los cuestionarios, y se enfocó en los cambios aplicados en ellos y en la corrección de los errores en la toma de muestras y las mediciones durante la prueba piloto. Este proceso se realizó en diferentes momentos del levantamiento, de acuerdo con los problemas y necesidades detectados por el personal de campo.

\section{Prueba piloto}

Al término de la primera fase de capacitación se realizó la prueba piloto en el estado de Morelos, con una duración de siete días, con la finalidad de evaluar los cuestionarios y la aplicación correcta y estandarizada de la toma de medidas somatométricas, de sangre y orina.

\section{Tratamiento de la información}

Esta etapa consistió en varios procesos manuales y automáticos de los que fueron objeto los cuestionarios, y comprendieron las etapas de empaquetado y envío, revisión y crítica, almacenamiento, captura, limpieza y construcción de base de datos.
Una vez recibidos los cuestionarios se sometieron a revisión y crítica exhaustivas por parte de un equipo centralizado y especialmente capacitado para identificar y, en su caso, corregir problemas en la información.

La captura se realizó mediante un sistema de captura inteligente, con el fin de reducir al mínimo errores en su digitación y obtener bases de datos limpias. Tras la captura se limpió la información en dos etapas: la primera consistió en establecer un diagnóstico general de la congruencia de la información y la segunda en aplicar tratamientos con el fin de garantizar relaciones lógicas y congruentes entre las variables de cada cuestionario, y entre los cuestionarios y los bancos de sueros.

\section{Referencias}

I. Secretaría de Salud. Dirección General de Epidemiología. Encuesta Nacional de Salud. México, DF: SSA, 1985.

2. Gutiérrez G, Sepúlveda-Amor J, Tapia-Conyer R. Encuesta Nacional Seroepidemiológica I: Diseño conceptual y metodología. Salud Publica Mex 1988;30:836-842.

3. Secretaría de Salud. Dirección General de Epidemiología. Encuesta Nacional de Nutrición. México DF: SSA, 1988.

4. Secretaría de Salud. Dirección General de Epidemiología. Encuesta Nacional de Adicciones. México DF: SSA, 1988.

5. Secretaría de Salud. Dirección General de Epidemiología. Encuesta Nacional de Cobertura de Vacunación. México DF: SSA, 1992.

6. Secretaría de Salud. Dirección General de Epidemiología. Encuesta Nacional de Enfermedades Crónicas. México DF: SSA, 1993.

7. Secretaría de Salud. Dirección General de Epidemiología. Encuesta Nacional de Adicciones II. México DF: SSA, 1993.

8. Tapia-Conyer R, Sepúlveda-Amor J, Solache-Alcaraz G. Encuesta Nacional Seroepidemiológica II: Diseño operativo. Salud Publica Mex 1994;30(6):843-852.

9. Secretaría de Salud. Dirección General de Epidemiología. Encuesta Nacional de Adicciones III. México DF: SSA, 1998.

10. Rivera-Domarco J, Shamah-Levy T,Villalpando-Hernández S. Encuesta Nacional de Nutrición 1999. Estado nutricio de niños y mujeres en México. Cuernavaca, Morelos, México: Instituto Nacional de Salud Pública, $200 \mathrm{I}$.

II. Frenk J. El concepto y la medición de accesibilidad. Salud Publica Mex 1985;27(5):438-453.

12.Andersen R.Access to medical care in the USA. Medical Care 1978;16(7):533-546.

13. Arredondo A. Modelos explicativos sobre la utilización de servicios de salud: revisión y análisis. Salud Publica Mex 1992;34:36-49.

14. Avedis D. The quality of care: how can it be assessed? JAMA 1988;260:1743-1748.

15. Cochran WG.Técnicas de muestreo. México: Continental, 1982:513.

16. Tinajero M, Eslava G. Calibración en muestreo: una aplicación a la Encuesta Nacional de Ingreso y Gasto en Hogares 1992 y 1996. Serie Monografías. México: IIMAS-UNAM, 2000;9(2I):II3.

17. Lee E, Forthofer R, Lorimer R.Analyzing complex survey data. California: SAGE Publications, 1989.

18. Norma Oficial Mexicana que establece los requisitos para la separación, envasado, almacenamiento, recolección, transporte, tratamiento y disposición final de los residuos peligrosos biológico-infecciosos que se generan en establecimientos que presten atención médica. NOM-087ECOL-1995; Secretaría del Medio Ambiente, Recursos Naturales y Pesca. Diario Oficial de la Federación, 12 de Junio de 1996. 